\title{
Chemical Characterization of Material Surfaces Using X-ray Photoelectron Spectroscopy (XPS): The Perfect Complement to Electron Microscopy Techniques
}

\author{
BR Strohmeier ${ }^{1}$, RG White ${ }^{2}$, TS Nunney ${ }^{2}, \mathrm{P} \mathrm{Mack}^{2}$, and AE Wright ${ }^{2}$ \\ ${ }^{1}$ Thermo Fisher Scientific, 5225 Verona Road, Madison, WI, 53711 USA \\ ${ }^{2}$ Thermo Fisher Scientific, Imberhorne Lane, East Grinstead, West Sussex, RH19 1UB \\ UK
}

Field emission scanning electron microscopy (FESEM) and scanning transmission electron microscopy (STEM) are valuable microscopic imaging tools for characterizing the morphology and structure of solid materials. When combined with energy dispersive X-ray spectroscopy (EDS), these techniques also provide elemental composition information on the near-surface and subsurface of materials up to several micrometers deep. However, EDS gives no detailed information regarding the chemical composition within the top few nanometers of the surface. The topmost surface chemistry of materials influences their interaction with other substances in the environment and thus affects important material properties such as adhesion strength, catalytic activity, corrosion resistance, susceptibility to oxidation, and other critical chemical and physical characteristics. Knowledge of surface chemistry is therefore crucial to the successful production and optimization of innumerable advanced materials used in modern devices such as coatings, ceramics, composites, metals, nanomaterials, polymers, semiconductors, and thin films, among others. Highly developed surface sensitive techniques are required to properly and fully characterize the surfaces of complex materials and specialized products, which cannot be accomplished with electron microscopy techniques alone.

X-ray Photoelectron Spectroscopy (XPS) is a qualitative and quantitative chemical analysis technique that probes only the outer few nanometers of the surface, thus enabling researchers to better understand what surface compositional properties influence optimum material performance [1]. XPS is capable of detecting all elements except for hydrogen and helium. XPS depth profiling, where XPS is combined with monatomic argon ion or cluster argon ion sputtering (i.e., surface etching), allows the identification of chemical variations from the topmost surface of solid materials (i.e., $\leq \sim 10 \mathrm{~nm}$ ) to hundreds of nanometers or more into the bulk, which facilitates the characterization of complex layered structures. XPS is the only analytical technique providing quantitative elemental and chemical state information with extremely high surface sensitivity, as well as in-depth, thus making XPS the technique of choice for comprehensively characterizing material surfaces and interfaces. So called "small spot" XPS allows the analysis of surface features ranging in size from a few tens to a few hundred micrometers. In addition to point analyses, XPS can, depending on the particular instrument used, produce linescans and chemical parallel images and/or scanned maps of surfaces with spatial resolutions of a few tens to just a few micrometers. Hence, XPS is the perfect complementary technique for materials characterization studies involving the use of electron microscopy techniques.

This presentation will include a brief review of the basic principles, capabilities, and advantages of XPS. A number of case studies will then be presented where XPS analyses were used in conjunction with electron microscopy techniques to characterize a variety of materials including: nanomaterials, multilayer thin film structures like solar cells and solar-control glass coatings, forensic trace evidence, and others. For example, Figure 1 shows a low energy $(30 \mathrm{keV})$ bright field STEM (BFSTEM) image of a sample of single-walled carbon nanotubes (SWCNTs) that contained a nickel/yttrium catalyst 
impurity. The BFSTEM image indicates the catalyst particles ranged in size between $\sim 5-20 \mathrm{~nm}$ and were mostly imbedded in amorphous carbon material (as indicated by EDS). The EDS map shown in Figure 1 indicated that the $\mathrm{Ni}$ and $\mathrm{Y}$ were closely associated with each other in the catalyst particles. However, EDS could not reveal any chemical information concerning the catalyst composition. XPS results shown in Figure 2 indicated that the $\mathrm{Ni}$ was present only as Ni metal whereas the $\mathrm{Y}$ was present as a mixture of $\mathrm{Y}$ metal and $\mathrm{Y}$ oxide $\left(\mathrm{Y}_{2} \mathrm{O}_{3}\right)$ based on the observed peak shapes and binding energies [2]. Since Ni metal is prone to oxidation, these results suggest that the catalyst particles consist of a $\mathrm{Ni}$ metal core covered and protected by a layer of Y metal that is in turn oxidized on the outer surface.

\section{References:}

[1] D Briggs and JT Grant, "Surface Analysis by Auger and X-ray Photoelectron Spectroscopy," (IM Publications, Chichester, West Sussex, UK) 2003.

[2] JF Moulder et al, "Handbook of X-ray Photoelectron Spectroscopy," (Perkin Elmer Corporation, Eden Prairie, MN, USA) 1992, p. 107.

[3] The authors acknowledge Dr. KL Bunker, RJ Lee Group, Inc., for obtaining the STEM image and EDS map of the SWCNT sample shown in Figure 1.
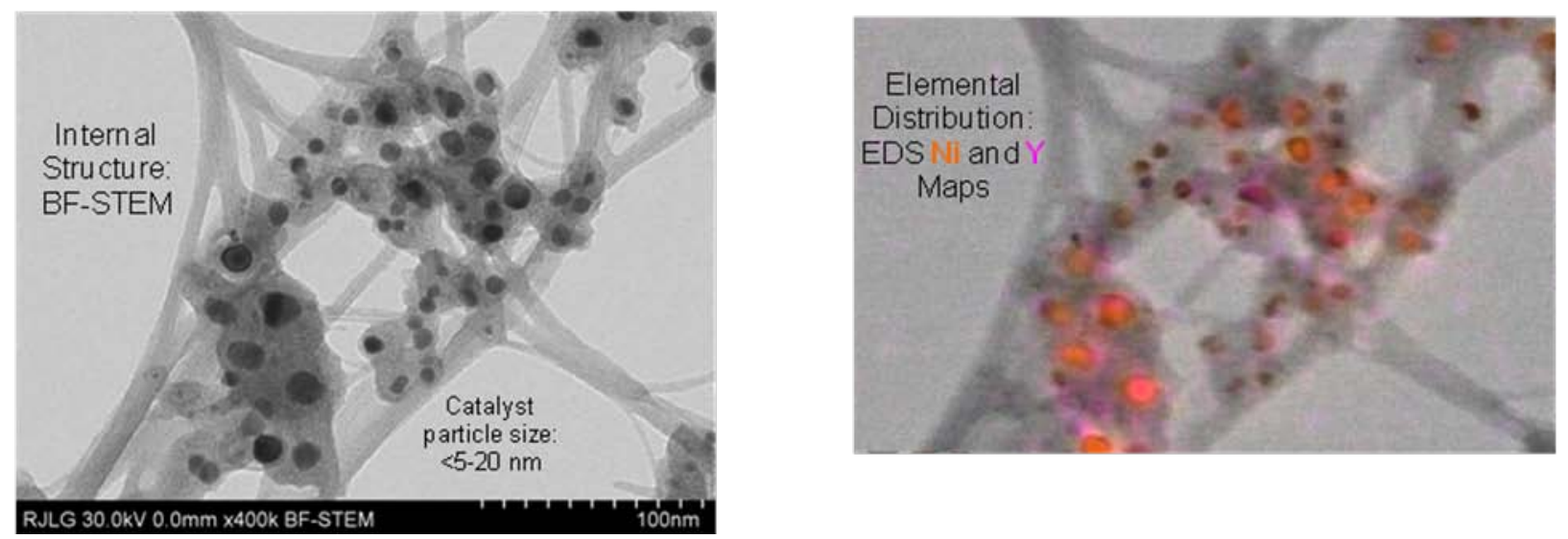

Figure 1. Bright field STEM image (left) and corresponding Ni (orange) and Y (purple) EDS maps (right) for SWCNTs having a Ni/Y catalyst impurity.
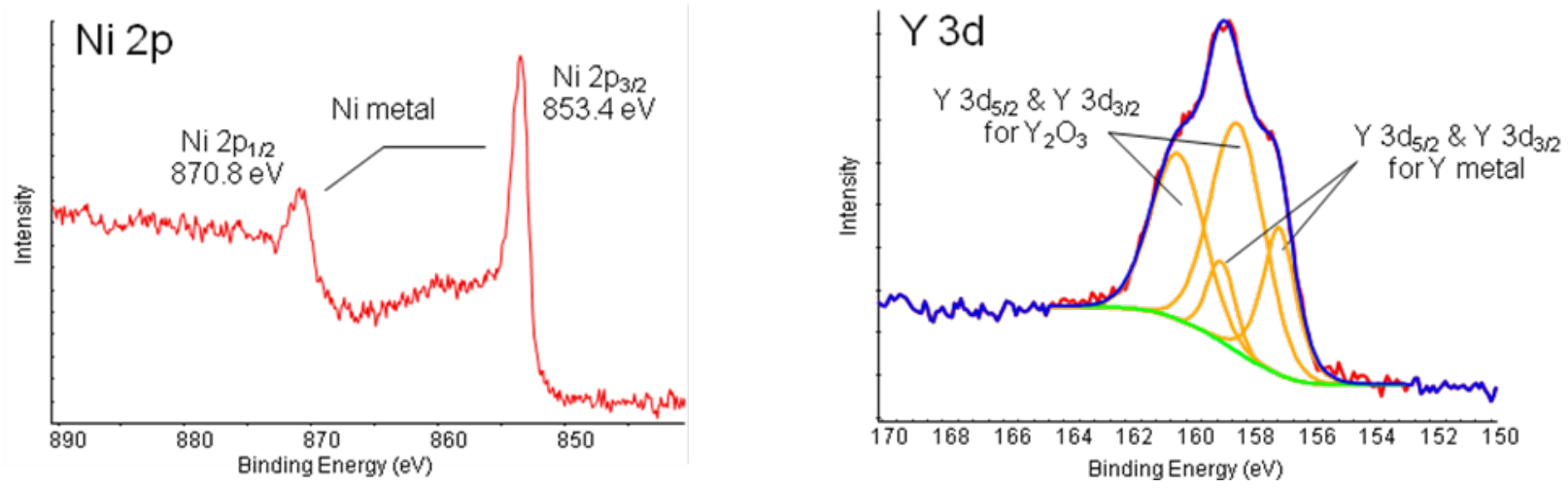

Figure 2. High resolution Ni 2p (left) and Y 3d (right) XPS spectra for SWCNTs having a Ni/Y catalyst impurity. The Y $3 \mathrm{~d}$ spectrum was fit with doublet peak components corresponding to $\mathrm{Y}$ metal and $\mathrm{Y}_{2} \mathrm{O}_{3}$. 